\title{
Pacifique Sud 2020-2021 Une saison délimitée par deux cyclones tropicaux très intenses
}

\author{
Corinne Thibord \\ Météo-France, Trappes
}

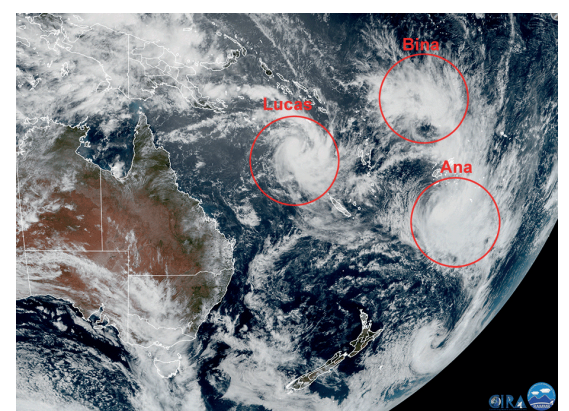

Vue satellitaire sur le bassin du Pacifique Sud le

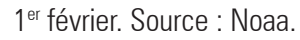

\section{Dossier réalisé d'après les sites Internet suivants :}

- Météo-France/Nouvelle-Calédonie http://www.meteo.nc/

- Bureau de la météorologie de l'Australie http://www.bom.gov.au/cyclone/

\section{- Australian Severe Weather} http://australiasevereweather.com/ cyclones/

\section{- CycloneXtrème}

http://www.cyclonextreme.com

- Colorado state university

http://tropical.atmos.colostate.edu

\section{- Wikipedia}

http://en.wikipedia.org/wiki/202021_South_Pacific_cyclone_season http://en.wikipedia.org/wiki/202021_Australian_region_cyclone_ season
C onséquence d'un épisode «la Niña » (océan plus froid que d'habitude à l'échelle du bassin Pacifique équatorial tout entier et zone de cyclogenèse déplacée vers le sud), la saison cyclonique 2020-2021 s'est révélée moins intense qu'à l'accoutumée. Contrairement à ce qui est observé habituellement dans le Pacifique Sud-Ouest lors d'une saison «la Niña», la zone de formation des cyclones ne s'est pas décalée plus à l'ouest que la normale : quatre phénomènes se sont formés près des côtes australiennes dont deux dans le golfe de Carpentarie (baptisés par le BoM) et quatre ont pris naissance entre le
Vanuatu et les Samoa (baptisés par le FMS). La saison est conforme à la moyenne obtenue sur les dix dernières années (tableau 1). Ce résultat de huit phénomènes pour la saison est un peu inférieur à la normale climatologique 1981-2010 qui est de neuf.

Sur les huit baptêmes de cette saison (tableau 2), trois ont évolué en cyclones tropicaux. Cette proportion de $37,5 \%$ est inférieure à celle obtenue sur les dix dernières années qui est de $46,3 \%$. Chacune des deux zones de responsabilité (FMS et BoM) comptabilise cette année un cyclone tropical très intense.

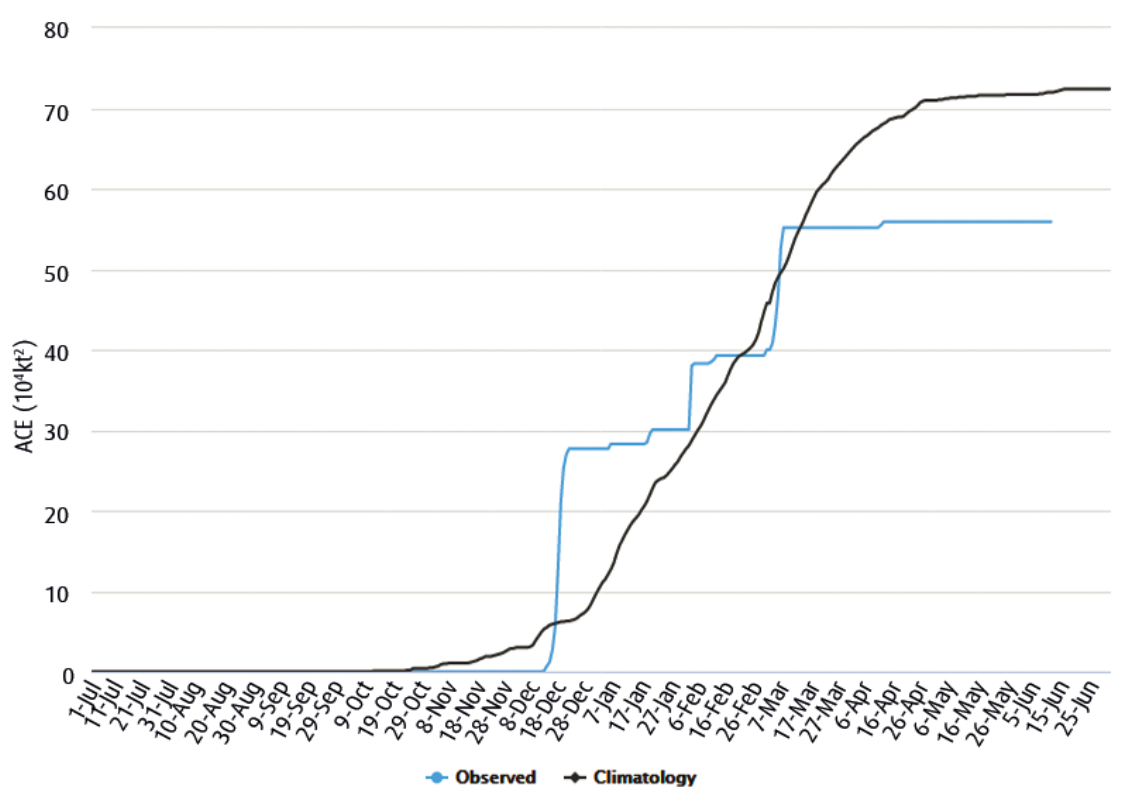

Figure 1. Évolution du cumul de l'ACE au cours de la saison 2020-2021 dans le Pacifique Sud (en bleu) comparée à la normale climatologique (en noir). Source : site du « Colorado State University ".

Tableau 1. Nombre de phénomènes baptisés (vent $\leq 34$ nœuds) sur le Pacifique Sud au cours de la dernière décennie.

\begin{tabular}{|lcccccccccccc|}
\hline Année & 2011 & 2012 & 2013 & 2014 & 2015 & 2016 & 2017 & 2018 & 2019 & 2020 & Moyenne \\
& 2012 & 2013 & $2014^{*}$ & $2015^{*}$ & 2016 & $2017^{*}$ & $2018^{*}$ & 2019 & 2020 & 2021 & \\
\hline Nombre & 4 & 8 & 11 & 9 & 8 & 6 & 8 & 9 & 9 & 8 & 8,0 \\
\hline
\end{tabular}

* Gillian est apparu et a séjourné dans le golfe de Carpentarie, puis dans l'océan Indien Sud-Est. II est comptabilisé dans les deux bassins.

** La tempête subtropicale Katie n'est pas comptabilisée dans ces statistiques.

*** Alfred pour 2016-2017 et Nora pour 2017-2018 évoluent à la limite des deux bassins, Pacifique Sud et océan Indien Sud-Est, dans le golfe de Carpentarie, ils sont comptabilisés dans ces statistiques pour le Pacifique Sud. 


\section{Gestion des phénomènes tropicaux dans le Pacifique Sud}

Le bassin cyclonique du Pacifique Sud se situe entre $135^{\circ} \mathrm{E}$ et $120^{\circ} \mathrm{O}$, englobant la côte est de l'Australie, la Nouvelle-Calédonie, la Nouvelle-Zélande, le Vanuatu, les îles Fidji, Tokelau, Wallis et Futuna, les îles Tonga et Samoa ainsi que les îles Cook et la Polynésie française. À l'ouest de $135^{\circ} \mathrm{E}$, il s'agit du bassin Océan Indien Sud-Est.

Sur ce bassin, différents centres météorologiques sont chargés de la surveillance et de l'émission des bulletins d'alerte cyclonique (voir figure). II s'agit essentiellement, de part et d'autre du méridien $160^{\circ} \mathrm{E}$, du CMRS (Centre météorologique régional spécialisé) Nandi (FMS, Fidji Meteorological Service) et du TCWC (Tropical Cyclone Warning Center) Brisbane (BoM, Australian Bureau of Meteorology). Chacun d'eux, FMS et BoM, établit une liste de noms pour les phénomènes qu'ils baptisent.

Les forces armées des États-Unis, par l'intermédiaire du Joint Typhoon Warning Centre (JTWC), surveillent également le bassin et diffusent des avertissements en faveur des intérêts américains.

Pour Météo-France, les centres de Nouvelle-Calédonie et de Wallis-et-Futuna participent à la mission de sécurité des personnes et des biens, mais n'ont pas de responsabilité cyclonique internationale. Statistiquement, la saison débute le 1er novembre et se termine le 30 avril, ce qui correspond à la période annuelle la plus propice aux systèmes cycloniques, avec un pic d'activité de mi-janvier à mi-mars et $50 \%$ des phénomènes nommés. En dehors de cette période de 6 mois ( $75 \%$ des saisons durent moins de 167 jours), le risque cyclonique, même s'il est relativement faible, n'est pas nul. Le CMRS Nandi attache un numéro et un suffixe $\mathrm{F}$ aux perturbations tropicales qui se

forment ou se déplacent dans le bassin, tandis que le JTWC désigne les cyclones tropicaux avec un numéro et un suffixe $P$.

Classification des ouragans. L'échelle Saffir-Simpson est employée pour la classification des ouragans et utilise, pour les vents soutenus, des moyennes calculées sur 1 minute.

\begin{tabular}{l|c|c}
$\begin{array}{l}\text { Nouvelle Calédonie / } \\
\text { Wallis et Futuna }\end{array}$ & $\begin{array}{c}\text { Vents soutenus } \\
\text { sur } \mathbf{1 0} \text { minutes (en nœuds) }\end{array}$ & $\begin{array}{c}\text { *Échelle australienne / Fidji } \\
\text { Dépression tropicale faible }\end{array}$ \\
\hline Dépression tropicale modérée & 34 à 47 & Perturbation tropicale \\
\hline Dépression tropicale forte & 48 à 63 & Catégorie 1 \\
\hline Cyclone tropical & 64 à 86 & Catégorie 2 \\
\hline Cyclone tropical intense & 87 à 107 & Catégorie 3 \\
\hline Cyclone tropical très intense & $\geq 108$ & Catégorie 4 \\
\hline
\end{tabular}

* * Pour l'échelle australienne, on parle de cyclone tropical pour les catégories 1 et 2 et de cyclone tropical intense à partir de la catégorie 3 .

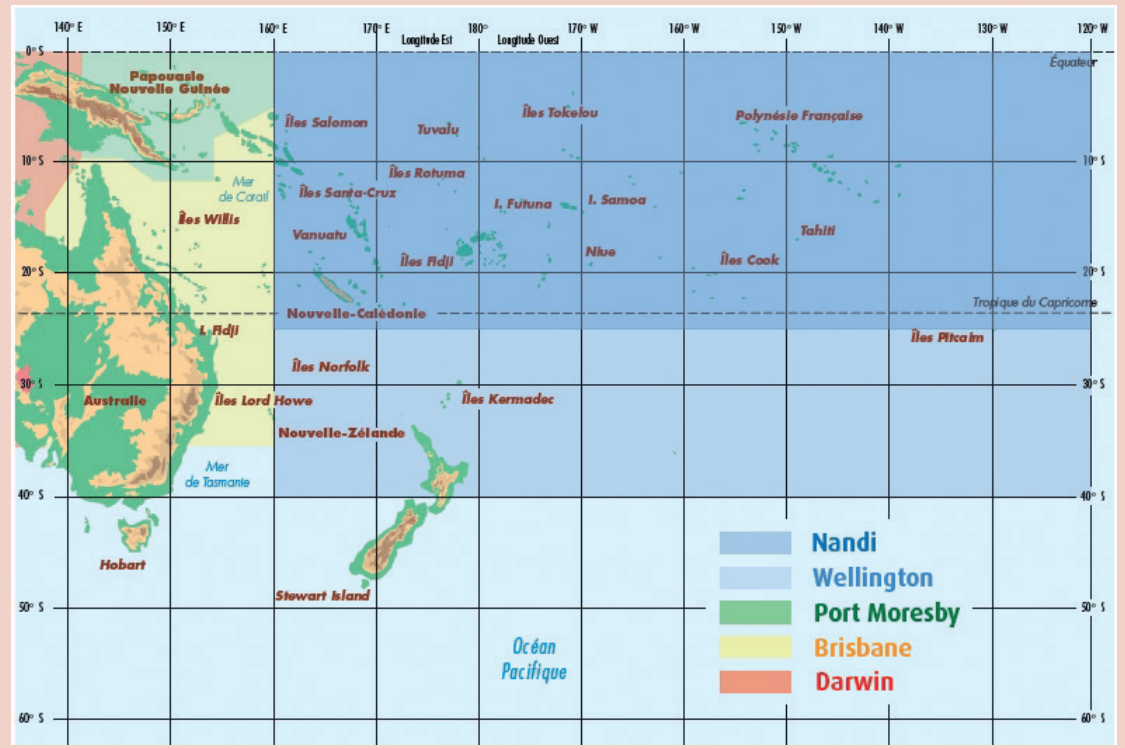

Secteurs de surveillance dans le Pacifique Sud.
Les cyclogenèses de sept des huit phénomènes sont concentrées sur la période couvrant la seconde moitié de décembre 2020 et le mois de janvier 2021. Niran, le phénomène qui a clos la saison, s'est dissipé le 7 mars 2021. La saison commencée tardivement avec un cyclone tropical très intense se termine précocement avec le même type de phénomène.

Pour la zone de surveillance de la Nouvelle-Calédonie, le nombre de quatre phénomènes observés au cours de la saison 2020-2021 est conforme à l'activité cyclonique attendue en situation « la Niña ». Seuls deux d'entre eux ont eu de réelles conséquences sur le pays : Lucas en février et Niran en mars.

En ce qui concerne l'énergie cyclonique accumulée (ACE), qui qualifie une saison en prenant en compte à la fois le nombre, la durée et l'intensité des épisodes qui la constituent, cette saison se situe en dessous de la moyenne concernant la période de référence de 1981-2010 (figure 1).

\section{La saison commence tardivement dans l'est du bassin}

Alors que la saison cyclonique dans ce bassin commence officiellement le ler novembre, la formation de la dépression tropicale $01 F$ n'est observée que le 8 décembre et n'atteint pas l'intensité suffisante pour être baptisée. C'est le 11 décembre que le FMS signale le développement des deux perturbations tropicales suivantes entrant dans la liste des phénomènes baptisés pour la saison.

D'abord noté comme la zone de basse pression $02 F$ au nord de Port-Vila au Vanuatu, le système se développe progressivement en absorbant la dépression tropicale $01 F$ les jours suivants. Ces deux minimums dépressionnaires fusionnés forment la dépression tropicale modérée Yasa au nord-est du Vanuatu, le 13 décembre. Soumis à des conditions très favorables, le phénomène se développe rapidement en cyclone tropical très intense. À son pic d'intensité, il génère des rafales estimées à près de $300 \mathrm{~km} / \mathrm{h}$. Il traverse les îles Fidji le 17 décembre, faisant plusieurs morts et des dégâts considérables, rappelant le passage du cyclone Winston en février 2016. Il décline 
ensuite rapidement en s'éloignant vers le sud. Yasa a maintenu le stade de cyclone très intense pendant plus de 36 heures.

« Le cyclone Yasa a été l'un des événements les plus intenses jamais enregistrés dans le Pacifique au cours des quarante dernières années. Les vents ont atteint 135 nœuds en moyenne, ce qui le placerait derrière Winston (150 nœuds en 2016) en deuxième position ex aequo avec Pam (2015) dans le classement des cyclones les plus violents. " Météo-France NouvelleCalédonie.

La zone de basse pression $03 F$ se forme quant à elle au sud des Samoa. Elle se renforce vers le nord des Tonga où elle est nommée Zazu, le 14 décembre. Elle atteint son pic d'intensité au stade de dépression tropicale forte le 15 décembre, en commençant à s'évacuer vers le sud-est, et génère alors des rafales à $140 \mathrm{~km} / \mathrm{h}$. Elle poursuit ensuite vers le sud, devenant une dépression extratropicale.

L'activité pluvieuse en décembre a été supérieure à la normale dans la région de Wallis-et-Futuna, du fait notamment des passages des cyclones Yasa et Zazu.

\section{L'activité se poursuit autour de la péninsule du cap York}

Un faible système de basse pression se développe dans le Territoire du Nord (Australie), le $1^{\text {er }}$ janvier. Il évolue le lendemain en dépression tropicale et se dirige ensuite vers le sud-est, à travers le golfe de Carpentarie, se déplaçant parallèlement à la côte sud du golfe. La dépression passe au-dessus de l'île Mornington le 3 janvier et le système est baptisé Imogen après s'être éloigné de l'île. Il s'intensifie légèrement au fur et à mesure de son approche de la côte. Touchant terre dans les six heures suivant son appellation, près de Karumba, il apporte de grandes vagues, de forts coups de vent, des marées anormalement hautes dépassant la plus haute marée astronomique, puis de fortes pluies et des inondations dans le nord du Queensland où il faiblit rapidement.

Au milieu du mois de janvier, un minimum dépressionnaire se développe

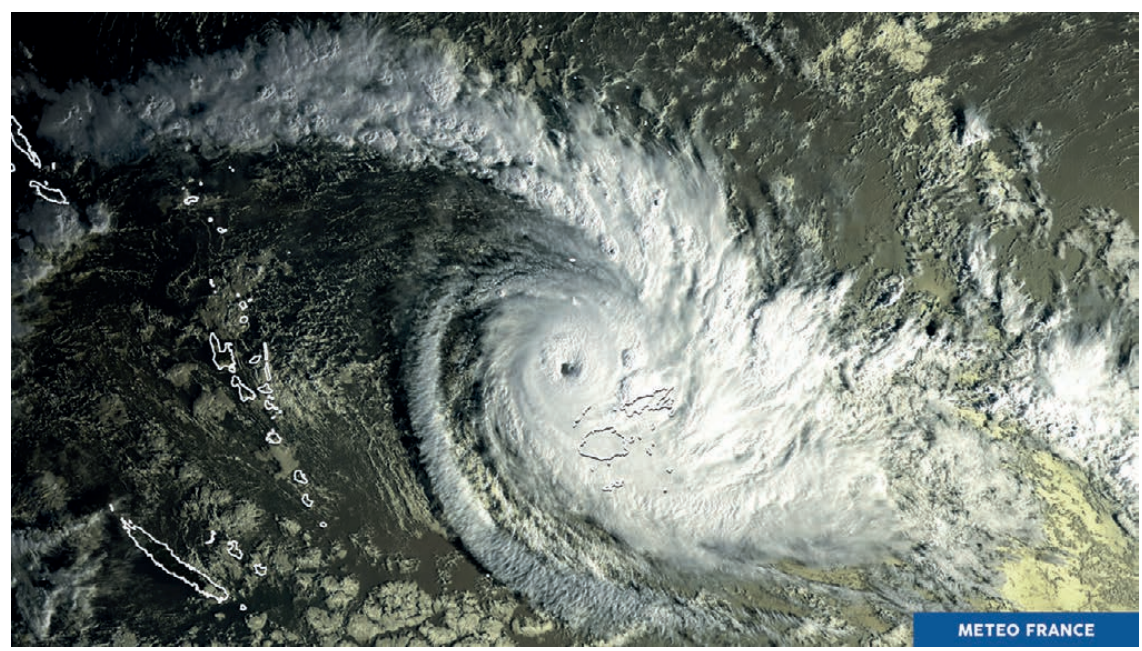

Yasa, un cyclone tropical de catégorie 5 sur l'échelle de Saffir-Simpson, se déchaîne depuis quelques jours sur l'océan Pacifique. Ce système tropical, I'un des plus puissants observés sur ce bassin, présente ici un œil bien ouvert dans lequel la pression atmosphérique chute à une valeur de $907 \mathrm{hPa}$ ! Autour de cette zone, les vents tourbillonnent à plus de $230 \mathrm{~km} / \mathrm{h}$, avec certaines rafales de l'ordre de $300 \mathrm{~km} / \mathrm{h}$. Ils s'accompagnent de vagues de près de 10 mètres de haut. Toute cette puissance destructrice est en train de s'abattre sur les Fidji. Image du satellite Himawari-8, le 16 décembre, à 19 h TU, publiée par Météo-France/CMS.

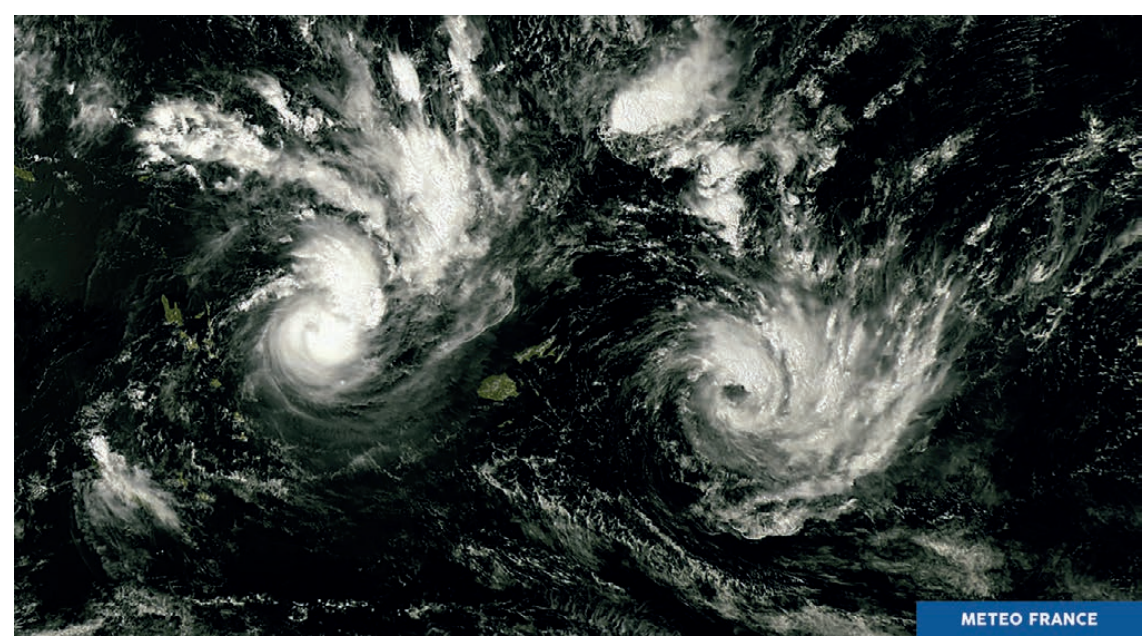

De gauche à droite, on retrouve les îles Vanuatu, le cyclone Yasa, les îles Fidji et la tempête tropicale Zazu. Le cyclone Yasa devrait prendre la direction des Fidji. Actuellement en catégorie 1, ce cyclone pourrait s'intensifier et passer en troisième catégorie. La trajectoire de Zazu, en revanche, devrait l'éloigner des Fidji. Image du satellite Himawari-8, le 14 décembre, à 0 h TU, publiée par Météo-France/CMS.

en mer de Corail, au large de la côte nord-est de la péninsule du cap York. Devenu dépression tropicale le 16 janvier, il se dirige vers le sud et se développe. Le système est baptisé Kimi le 17 janvier. Kimi continue de se déplacer vers le sud, presque parallèlement à la côte nord du Queensland. Les estimations de l'intensité du système, à l'aide d'images satellitaires, indiquent qu'il pourrait avoir atteint l'intensité de la catégorie 2 pendant une courte période. Kimi poursuit sa trajectoire vers le sud en perdant de son intensité face à des conditions défavorables, puis amorce un demi-tour vers le nord-ouest, près de la côte, tôt le 19 janvier, sans se développer, avec peu ou pas d'impact sur le continent.

\section{Retour vers les îles Fidji}

Un peu plus d'un mois après Yasa, une dépression se forme le 27 janvier dans un axe de basses pressions à l'est du Vanuatu. Elle est nommée Ana le 30 janvier au nord-ouest de Fidji, qu'elle atteint au stade de dépression tropicale forte entre le 30 et le 31 janvier. C'est le deuxième impact direct de la saison cyclonique pour le pays. Des quartiers sont inondés, des routes détruites et plusieurs personnes sont portées disparues. Ana devient brièvement cyclone tropical dans la nuit du 31 janvier en s'éloignant vers le sud, puis perd en intensité rapidement et dégénère en dépression subtropicale le $1^{\text {er }}$ février. 
Sur les pas de Ana, un autre phénomène s'approche des Fidji. Cette nouvelle dépression qui s'est développée au nord du Vanuatu se dirige vers Viti Levu et Vanua Levu (les deux grandes îles fidjiennes). Elle évolue au stade de dépression tropicale modérée le 30 janvier pour être baptisée Bina. Elle se maintient à cette intensité durant une journée au nord-est des Fidji, avant de traverser celles-ci au stade de dépression tropicale faible, à la suite d'une désorganisation rapide, le $1^{\text {er }}$ février.

\section{Impacts sur la Nouvelle-Calédonie pour la fin de saison}

Alimentée par un flux de mousson, une perturbation tropicale est observée par le centre d'alerte cyclonique de Brisbane, le 26 janvier, au nord du continent australien dans le golfe de Carpentarie. Le système traverse la péninsule du cap York, occasionnant des pluies abondantes, et progresse vers l'est au-dessus des eaux chaudes de la mer de Corail qu'il atteint le 29 janvier. La perturbation s'intensifie, devenant une dépression tropicale faible le 30 janvier. La dépression se situe à plus de $300 \mathrm{~km}$ à l'est des côtes australiennes et à $400 \mathrm{~km}$ au sud de la Papouasie-Nouvelle-Guinée. Elle poursuit son périple vers l'est tout en se creusant. C'est le 31 janvier que, devenue dépression tropicale modérée, elle est baptisée Lucas par les météorologues de Brisbane. Quelques heures plus tard, le phénomène devient une dépression tropicale forte. Le $1^{\text {er }}$ février, la dépression franchit le méridien $160^{\circ} \mathrm{E}$ et le CMRS de Fidji prend en charge son suivi. Le lendemain, Lucas passe à environ $300 \mathrm{~km}$ au nord de l'archipel des Bélep et atteint son pic d'intensité, puis prend la direction des îles Loyauté. Son intensité décroît, en raison d'une zone de vents forts en altitude. Lucas est déclassée au rang de dépression tropicale modérée, mais son activité pluvio-orageuse se renforce brusquement et un déluge de pluies s'abat sur Ouvéa (figure 2). Quant à l'île de Lifou, sa côte est, sur la trajectoire de Lucas, est affectée par des vents violents de $170 \mathrm{~km} / \mathrm{h}$, un nouveau record depuis le passage de Donna (162 km/h en 2017).

Le 3 février, Lucas gagne la pointe sud de la Nouvelle-Calédonie où les vents et les pluies les plus intenses ont devancé de plusieurs heures l'arrivée du centre de la dépression. À ce stade, la

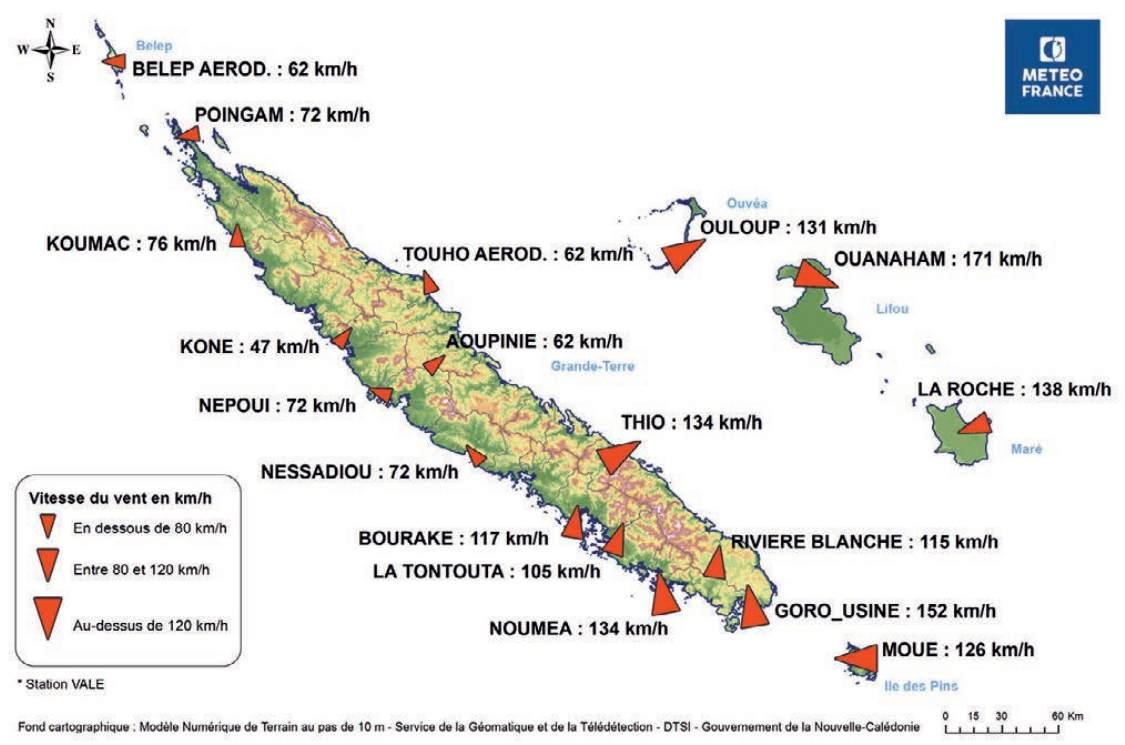

Figure 2. Rafales maximales mesurées entre le $1^{\text {er }}$ et le 4 février 2021. Source: Météo-France Nouvelle-Calédonie.

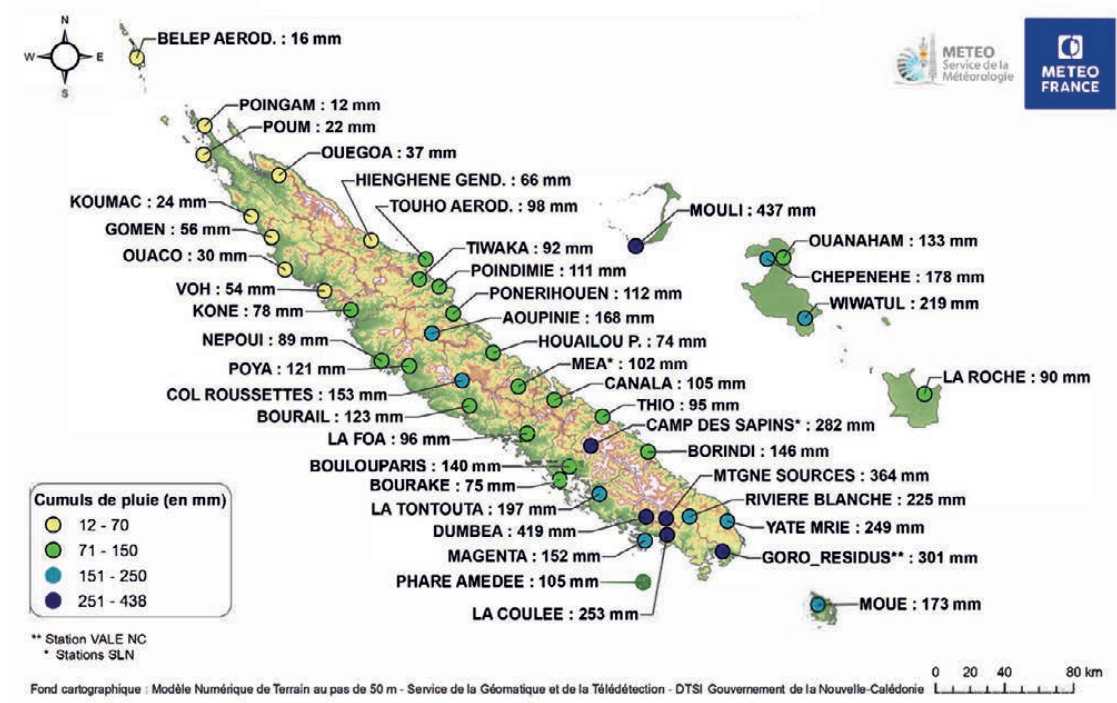

Figure 3. Cumuls de précipitations en 2 jours du 2 février 2021 à 5 h au 4 février 2021 à 5 h (heures locales). Source : Météo-France Nouvelle-Calédonie.

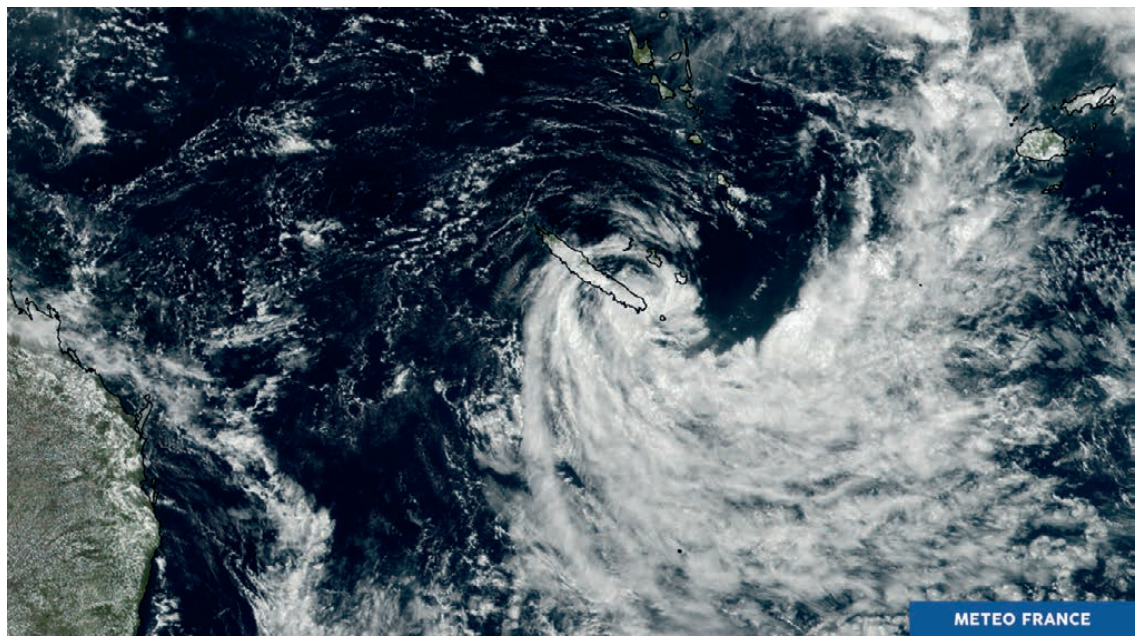

Dans l'océan Pacifique, en mer de Corail, le cyclone Lucas s'est atténué et est passé au stade de tempête tropicale. En Nouvelle-Calédonie, lors des observations de $6 \mathrm{~h}$ TU, il a été relevé en vent instantané : $80 \mathrm{~km} / \mathrm{h}$ à l'île des Pins et à Goro, $75 \mathrm{~km} / \mathrm{h}$ au phare Amédée. Les cumuls de pluie sont importants avec $430 \mathrm{~mm}$ à Ouvéa, $150 \mathrm{~mm}$ à Lifou, 200 à $300 \mathrm{~mm}$ sur le relief sud de la Grande Terre, 50 à $80 \mathrm{~mm}$ sur la moitié nord de la Grande Terre. Les pluies ont causé de nombreuses inondations sur le sud du territoire. Image du satellite Himawari-8, le 3 février, publiée par Météo-France/CMS. 
progression du phénomène stagne, engendrant un cumul très important de précipitations (figure 3). Le lendemain, 4 février, Lucas s'éloigne lentement au sud-ouest du pays avec un mouvement en zigzag et son intensité décline.

Après une pause d'un mois, l'activité cyclonique reprend avec un minimum dépressionnaire qui commence à se développer au large de la côte nord du Queensland, en mer de Corail, le 27 février. Stationnaire pendant plusieurs jours et bénéficiant de conditions favorables à son renforcement, ce minimum se creuse progressivement. Il se structure en dépression tropicale modérée le 2 mars. Le phénomène est alors baptisé Niran à environ $260 \mathrm{~km}$ de la côte nord du Queensland. Niran continue de se déplacer lentement pendant les deux jours suivants tout en s'intensifiant progressivement pour devenir une dépression tropicale forte. C'est à partir du 4 mars que Niran amorce sa trajectoire vers l'est-sud-est qui va le conduire vers la Nouvelle-Calédonie. Il se déplace très rapidement tout en se renforçant. Le système devient un cyclone tropical très intense le 5 mars, juste avant de franchir la frontière de la région est $\left(160^{\circ} \mathrm{E}\right)$. Il se situe alors à $480 \mathrm{~km}$ dans l'ouest-nord-ouest de Bélep. Le 6 mars, il accélère et longe la côte ouest

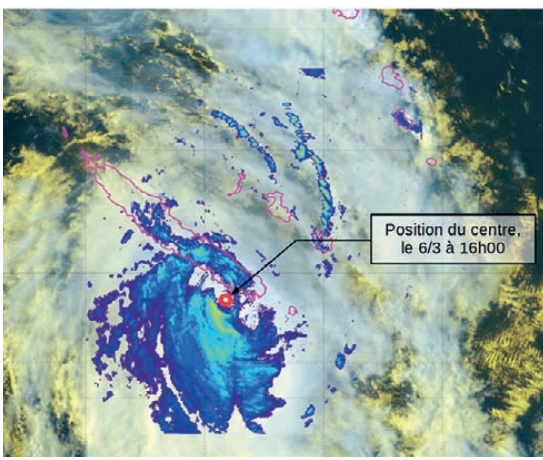

Figure 4. Niran le 6 mars 2021 à 16 h locale, lors de son passage au plus près du territoire. Image en canal visible issue du satellite Himawari-8 et réflectivité radar (précipitations). Source Météo-France Nouvelle-Calédonie. de la Nouvelle-Calédonie en perdant de son intensité et frôle le sud-ouest de la Grande Terre. Il passe à environ $20 \mathrm{~km}$ de Nouméa. Se déplaçant rapidement, il n'impacte le territoire que peu de temps. Toutefois, une mer déchaînée associée à une marée de tempête élevée et des vents violents $(150$ à $200 \mathrm{~km} / \mathrm{h})$

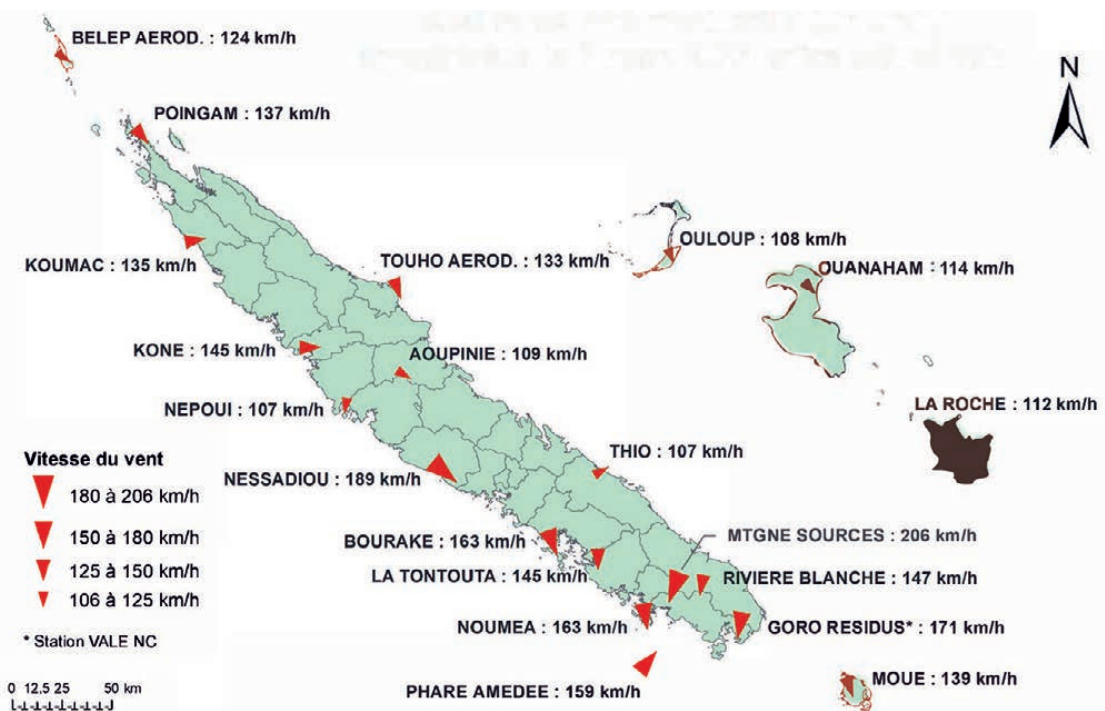

Figure 5. Rafales maximales de vents mesurées lors du passage du cyclone Niran le 6 mars 2021. Source : Météo-France Nouvelle-Calédonie.

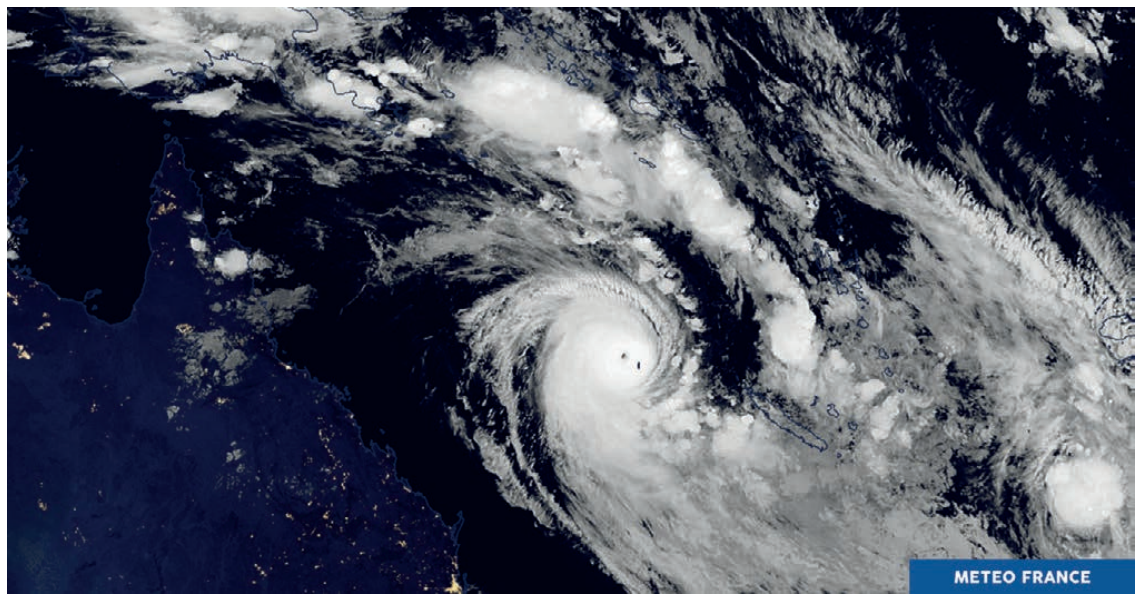

Le cyclone tropical intense Niran a atteint la catégorie 5 sur l'échelle de Saffir-Simpson. Les vents les plus forts près du centre sont de l'ordre de $205 \mathrm{~km} / \mathrm{h}$ avec des rafales à $285 \mathrm{~km} / \mathrm{h}$. La pression au centre est estimée à $944 \mathrm{hPa}$. II se rapproche de la Nouvelle-Calédonie plongée dans la nuit. Des fortes pluies, un vent très violent et une mer dangereuse sont attendus. L'alerte cyclonique est déclenchée. Image du satellite Himawari-8, le 5 mars, publiée par Météo-France/CMS.

Tableau 2. Dépressions tropicales et cyclones dans le Pacifique Sud en 2020-2021.

\begin{tabular}{|c|c|c|c|c|c|c|}
\hline Numéro & Nom & $\begin{array}{c}\text { Classification } \\
\text { Nouvelle-Calédonie }\end{array}$ & $\begin{array}{c}\text { Échelle } \\
\text { australienne }\end{array}$ & $\begin{array}{l}\text { Début } \\
\text { de période }\end{array}$ & $\begin{array}{c}\text { Fin } \\
\text { de période }\end{array}$ & $\begin{array}{l}\text { Vents maximum } \\
\text { soutenus sur } 10 \text { minutes } \\
\text { en nœuds (date) }\end{array}$ \\
\hline 1 & Yoso & Cyclone tropical très intense & 5 & $13 / 12 / 2020$ & $20 / 12 / 2020$ & $125(16 / 12 / 2020)$ \\
\hline 2 & Zozu & Dépression tropicale forte & 2 & $13 / 12 / 2020$ & $16 / 12 / 2020$ & $50(16 / 12 / 2020)$ \\
\hline 3 & Imogen & Dépression tropicale modérée & 1 & $01 / 01 / 2021$ & $06 / 01 / 2021$ & $45(03 / 01 / 2021)$ \\
\hline 4 & Kimi & Dépression tropicale forte & 2 & $16 / 01 / 2021$ & $19 / 01 / 2021$ & $50(17-18 / 01 / 2021)$ \\
\hline 5 & Ano & Cyclone tropical & 3 & $27 / 01 / 2021$ & $01 / 02 / 2021$ & $65(31 / 01 / 2021)$ \\
\hline 6 & Bino & Dépression tropicale modérée & 1 & $29 / 01 / 2021$ & $01 / 02 / 2021$ & $40(31 / 01 / 2021)$ \\
\hline 7 & Lucos & Dépression tropicale forte & 2 & $26 / 01 / 2021$ & $05 / 02 / 2021$ & $55(01 / 02 / 2021)$ \\
\hline 8 & Niron & Cyclone tropical très intense & 5 & $01 / 03 / 2021$ & $07 / 03 / 2021$ & $110(05 / 03 / 2021)$ \\
\hline
\end{tabular}

